\title{
CAD-CAM PRESCRIPTION AND DESIGN ITEMS OF CUSTOM MADE
} MEDICAL FOOTWEAR SOLES

\author{
DANIEL PETCU \\ INCDTP - Division Leather and Footwear Research Institute, 93 Ion Minulescu St, Bucharest, \\ Romania,icpi@icpi.ro
}

\begin{abstract}
Custom made medical footwear is a prescription-based medical device. In order for the endproduct to achieve the expected therapeutic effects, several conditions have to be fulfilled, such as: diagnosis and assessment of pathology and establishing the treatment goals, knowledge of the prescription items and of those technical characteristics of the product that allow to achieve these goals, the existence of flexible technologies enabling the design and practical execution of the prescription in the shortest possible time in terms of economic efficiency. In the absence of these factors, achieving the goals of the conservative treatment is difficult and frequently leads to failure. The present article proposes a review of the prescription parameters of custom made medical footwear soles and presentation of CAD-CAM solutions for their design. For this purpose, some sole prescription parameters were transposed into a virtual model ready for execution on automatic milling machines. CAD-CAM technologies enable the flexibilization of the custom made footwear sole design and manufacture processes and reduces the waiting times of patients The implementation of these technologies requires significant investments for their purchase and for training medical specialists and technicians involved in establishing and implementing the treatment.
\end{abstract}

Keywords: soles, medical footwear, CAD-CAM

\section{INTRODUCTION}

Custom-made medical footwear is a prescription based medical device. Custommade medical devices refer to "any device specifically made in accordance with a duly qualified medical practitioner's written prescription which gives, under his responsibility, specific design characteristics and is intended for the sole use of a particular patient. The abovementioned prescription may also be made out by any other person authorized by virtue of his professional qualifications to do so." (Directive 93/42/EEC). Seen from this perspective, the medical prescription must include the "constructive characteristics" of the used medical device having a therapeutic role in the conservative treatment of foot pathomechanics.

The medical literature lists a number of therapeutic effects and necessary prescription parameters of the medical devices used in the implementation of conservative treatment of foot pathomechanics. In this regard, as a pathology, therapeutic effects and specific design features for medical footwear soles include:

- pathologies:

- hallux rigidus or hallux limitus, metatarsalgia, healed plantar ulcerations, pes planus or foot with medially deviated subtalar joint, pes cavus or foot with laterally deviated subtalar joint, minor pains in ankle joint, blocked ankle joint, diabetic foot (Nawoczenski et al., 1988; Praet and Louwerens, 2003), rheumatoid arthritis foot (Cho et al., 2009), knee injuries, etc.

- therapeutic effects:

- modification of the range of motion in the angle joint (Petcu et al., 2013), decrease of the pressures from the distal metatarsal heads ulcerations area (Nawoczenski et al., 1988; Mueller, 1999), decrease of the supporting time on the affected joints, influencing the supinator or pronator moments through the modification of the ground reaction 
force moment arm relative to the anatomical joints of interest, modification of shock absorbing properties, etc. As main adverse effects we can note an increase of the postural instability as a result of sole height, hardness of the sole material and decrease of the contact surface with the supporting surface (Hijmans et al., 2007) or decrease of the impact shock absorbing properties and increase of the energetic consumption as a result of pronounced sole wearing (Saito et al., 2007).

- constructive characteristics / prescription parameters:

- the shape of the superior longitudinal sole profile and of superior surface will influence the way in which the foot will stay in contact with the sole. Together with the inferior longitudinal profile, foot stability inside the shoes, body posture and pressure distribution at the plantar foot surface could be influenced (Dananberg, 1998),

- the shape of the inferior longitudinal sole profile and of bottom surface. The effect of this type of profile is best exemplified in the case of the rocker sole (Hutchins, 2009). In this way, the main constructive characteristics which can be considered prescription parameters are: sole's rocker profile, proximal and distal contact lines with supporting surface, the orientation of these lines or of the inferior profile relative to the walking direction (Petcu et al., 2013), sole's height in different areas, medial or lateral extension of the distal area of the sole's heel with the purpose of influencing the stability or the pronator and supinator moments around the anatomical joint axis of interest (known as medial or lateral "Thomas heel"),

- the sole's lateral flange. This could be applied on different areas as the heel area or entire lateral area. When this flange is applied on both medial and lateral sides it will generate a trapezoidal shape of the sole section,

- the physico-mechanical properties of the soles such as the hardness of the used material or the properties which are defining the slipping phenomenon relative to the supporting surface (friction coefficient).

It should be mentioned that these constructive characteristics and their link with the therapeutic effect should be known by the medical device prescription specialist. They should be included in the prescription file which is the communication base between diverse categories of specialists involved in the conservation treatment. Knowledge of these constructive characteristics by the medical specialist will allow him to select the optimal sole shape in the case of a prefabricated product or an efficient communication with the technical specialist involved in the design and their practical manufacture in the case of custom-made devices. It should be mentioned that, in general, in Romania, these design parameters are not known to the medical specialists, the medical prescription being limited to an indication such as "orthopedic footwear". Meanwhile, in the case of technical specialists there is no knowledge of the potential biomechanical effect which can be created by a specific technical characteristic of the sole at the level of the musculoskeletal system. Another important aspect is the sole's design and manufacture process flexibility. In this respect it is worth mentioning the practical difficulty of manufacturing a footwear sole with a rocker profile oriented relative to the walking direction and not to the longitudinal foot axis. The use of 3D modeling software solutions with automatic milling technologies or, more recently, 3D printing process allows to maximize the flexibility of the design and manufacture process of soles, facilitating practical implementation of the prescriptive features otherwise very difficult to achieve practically. 
ICAMS $2016-6^{\text {th }}$ International Conference on Advanced Materials and Systems

\section{METHOD}

The modern design of medical footwear soles could be done based on many 3D modeling software solutions. The purpose of this paper is to exemplify one working method which can be used in the virtual modeling of the custom-made medical footwear. In this respect it is necessary first to know:

- the prescription parameters / constructive characteristics of the medical device as indicated by the medical specialist,

- the design and 3D modeling methods of the prescription parameters. This requires knowledge of generic operations that allow shaping lines, surfaces or volumes (for example extrusion lines or surfaces, cutting volumes, etc.),

- how these design methods can be put into practice through specific functions of available 3D modeling software solutions.

The software used to exemplify the work method was PowerSHAPE 2016 from Delcam company.

\section{RESULTS}

In this work a simulation was carried out for designing a rocker sole. In describing the design process only the generic names of the performed operations will be indicated and not the specific features or functions of the chosen software solution. This approach is justified by the fact that an operation can be performed using different functions or commands. How they are selected depends only on the ability and knowledge of the designer.

The selected prescription parameters for this simulation were as follows:

1. the angle of the longitudinal foot axis with walking direction, $a=12^{\circ}$ (Figure 1a),

2. footwear last's heel height of $65 \mathrm{~mm}$ (Figure 1b). In the absence of the virtual last, the longitudinal profile of the last bottom and its orientation relative to the prescribed heel height can be indicated,

3. the rocker profile as indicated in prescription, (Figure 1c). In this example, the longitudinal rocker profile corresponds to an antero-posterior rocker profile where the contact with supporting surface is represented by a line. In this situation the proximal and distal contact lines are confounded,

4. the orientation of the inferior longitudinal profile of the sole is parallel with walking direction. This option facilitates walking in the forward direction in the case of the feet having the longitudinal axis (the heel-toe axis) at a higher angle with walking direction than the normal one (approximately 7 degrees),

5. the lateral surface of the sole is vertical.

The main basic steps need to be taken are:

1. the virtual last is oriented in a such way as the longitudinal last's axis (the heel-toe axis) is making the prescribed angle, "a", with walking direction (Figure 1a),

2. an extrusion, $\mathrm{E}$, of the last's plantar surface (bottom last) is made in order for the superior sole's surface to be obtained, the extrusion height being equal with the sum of the last insole thickness and upper thickness in the lasting area. ("E" - Figure 1b),

3. the rocker profile is designed in the last' sagittal plane (Figure 1b), 


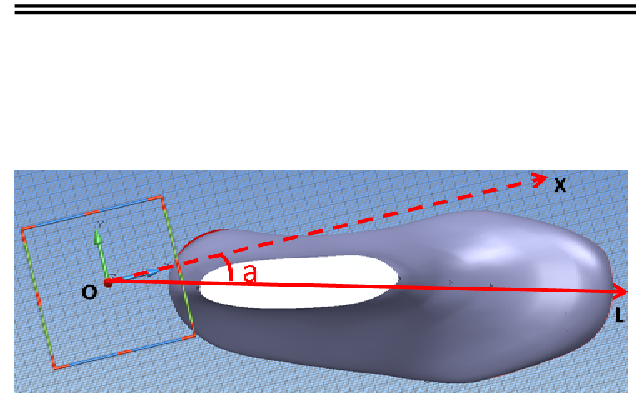

a)

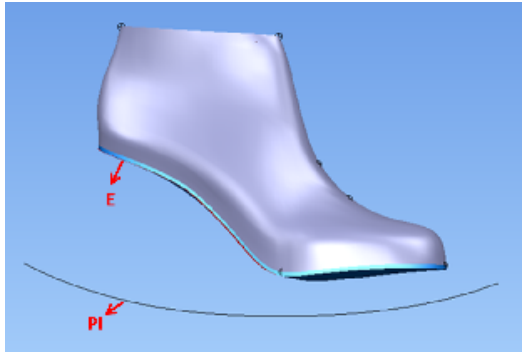

b)

Figure 1. Last orientation and design of the inferior profile: a) the last's longitudinal axis orientation, OL, relative to the walking direction, Ox, b) the design of the inferior profile, $\mathrm{Pl}$, relative to the last's oriented position

4. the rocker profile's extrusion in the transversal (horizontal) plane is made. Its orientation relative to walking direction is presented in Figure 2a,

5. the vertical extrusion of the superior outline of the sole obtained in step no. 1 is made (Figure $2 b$ ),

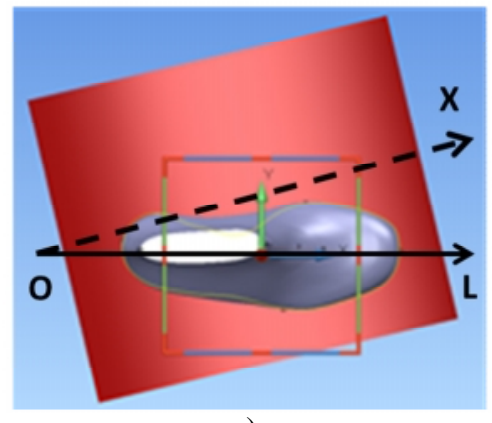

a)

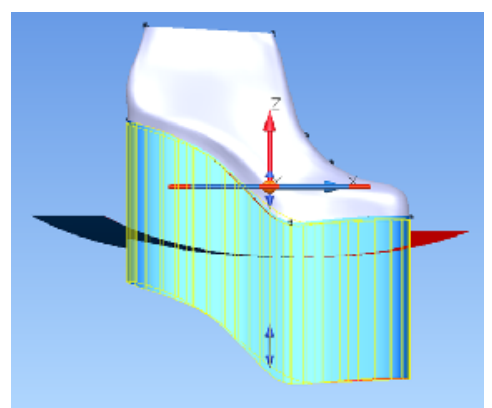

b)

Figure 2. Sole design: a) inferior profile extrusion, b) superior surface extrusion

6. the lateral sole surface is obtained by cutting the vertically extruded surface with the horizontally extruded rocker profile (Figure 3a).

The extrusion options allow the design of soles having oblique or vertical walls. These options are useful in two situations:

- when the aim is to design a sole with a larger inferior surface area than the last's bottom area, in order for the footwear stability to be increased (the transversal section having a trapezoidal shape),

- when the aim is to modify the pronatory or supinatory moments through the modification of the reaction force moment arm relative to the anatomical joint axis of interest. In this case, a lateral (for the pronatory moment modification) or medial (for the supinatory moment modification) inclined transversal profile will be created. 
ICAMS $2016-6^{\text {th }}$ International Conference on Advanced Materials and Systems

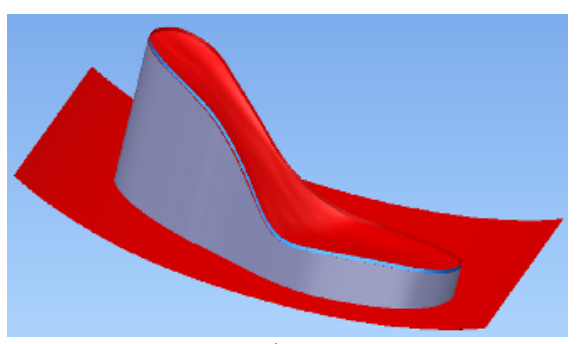

a)

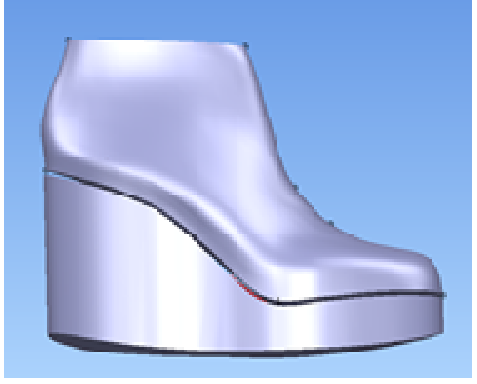

b)

Figure 3. Rocker sole: a) cutting of the extruded volume, b) the final sole shape and the last

7. the superior surface of the sole is obtained based on the outline of the surface resulted through the extrusion of the last's bottom (step no. 2),

8. the inferior surface of the sole corresponding to the wearing area is obtained using the outline resulted through the intersection of the lateral surface with the surface obtained through the inferior longitudinal profile's extrusion,

9. the surface defining the sole's volume is obtained through the union of all three anterior designed surfaces: superior plantar surface, lateral surface and inferior surface (wearing area),

10. for the automated milling or printing manufacture the designed virtual sole's volume is exported in a format which can be recognised by the CAM (Computer Aided Manufacture) software (.stl format for example).

The final shape of the sole and the last shape are presented in figure no.3b. The space created through the extrusion of the plantar surface ("E", step no. 2) can be seen, determined by the last insole and upper thickness.

\section{CONCLUSIONS}

Using CAD-CAM technologies allows the flexibilization of the designing and manufacturing process of the medical footwear soles and the reduction of the patient's waiting time. New prescription / constructive characteristics could be introduced and easily manufactured.

The use of these technologies requires significant investments for the acquisition and especially for the training of the medical and technical specialists involved in the treatment prescription and implementation processes.

In order for the final product to produce the estimated therapeutic effect, several conditions must be met, such as: the diagnosis and the evaluation of the pathology and establishing the treatment's objectives, knowledge of the prescription elements and of those technical characteristics of the product that will achieve the treatment's objectives, availability of flexible technologies which will allow the design and manufacture of the prescription in a shorter time and with economical efficiency. In the absence of these factors, achieving the conservative treatment's objectives is difficult, leading, not infrequently, to failure. 


\section{REFERENCES}

Cho, N.S. et al., (2009), "Randomized controlled trial for clinical effects of varying types of insoles combined with specialized shoes in patients with rheumatoid arthritis of the foot", Clinical Rehabilitation, 23, 512521.

Dananberg, H. (1998), “Comfortable High Heel Shoe”, US Patent 5782015, Jul. 21.

Hijmans, J.M., Geertzen, J.H.B., Dijkstra, P.U., and Postema, K. (2007), "A Systematic Review of the Effects of Shoes and Other Ankle or Foot Appliances on Balance in Older People and People with Peripheral Nervous System Disorders" Gait \& Posture, 25(2) 316-23, doi:10.1016/j.gaitpost.2006.03.010.

Hutchins, S., Bowker, P., Geary, N., and Richards, J. (2009), "The Biomechanics and Clinical Efficacy of Footwear Adapted with Rocker profiles-Evidence in the Literature", The Foot, 19(3), 165-70, doi:10.1016/j.foot.2009.01.001

Mueller, M.J. (1999), "Application of plantar pressure assessment in footwear and insert design", The Journal of Orthopaedic and Sports Physical Therapy 29, 747-755.

Nawoczenski, D.A., Birke, J.A., Coleman, W.C. (1988), "Effect of rocker sole design on plantar forefoot pressures", Journal of the American Podiatric Medical Association, 78, 455-460.

Petcu, D., Bucur, D., Karavana, H.A., Nistor, M., Pantazi, M., Georgescu, M. (2013), "The influence of the rocker sole profile's orientation on the sagittal ankle range of motion", The 4th IEEE International Conference on E-Health and Bioengineering - EHB 2013, Grigore T. Popa University of Medicine and Pharmacy, Iasi, Romania, November 21-23, 2013.

Praet, F.E., and Louwerens, J.K. (2003), "The Influence of Shoe Design on Plantar Pressures in Neuropathic Feet", Diabetes Care, 26(2), 441-445.

Saito, S., Muraki, S., and Tochihara, Y. (2007), "Effects of Worn-Out Soles on Lower Limb Stability, Shock Absorption and Energy Cost during Prolonged Walking", Journal of Physiological Anthropology, 26(5), 521-526, DOI: 10.2114/jpa2.26.521.

*** Council Directive 93/42/EEC of 14 June 1993 concerning medical devices, http://eur-lex.europa.eu/legalcontent/EN/TXT/?uri=CELEX:31993L0042.

*** Delcam PowerShape Documentation, http://www.delcam.com/software/powershape 